Between the 'Lines

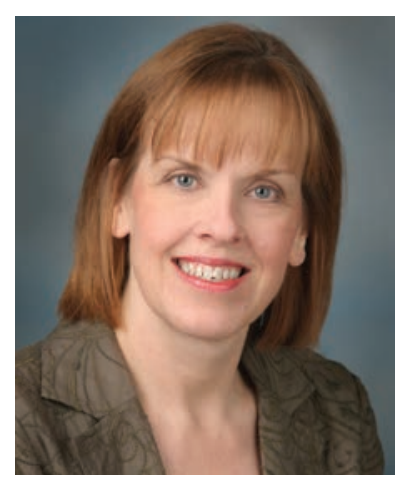

Therese B. Bevers, MD

Therese B. Bevers, MD, is professor of Clinical Cancer Prevention and Medical Director of the Cancer Prevention Center and Prevention Outreach Programs at MD Anderson Cancer Center.

In her role as Medical Director, Dr. Bevers has overseen the growth and program development of the Cancer Prevention Center-the first comprehensive clinical cancer prevention service program in the country-since its opening in 1996 Her clinical and research interests are in the area of breast cancer prevention, screening, diagnosis, and survivorship. She was the MD Anderson co-principal investigator (PI) on the groundbreaking Breast Cancer Prevention Trial and the STAR trial. She is currently investigating the potential role of vitamin $D$ in breast cancer prevention. Dr. Bevers chairs the NCCN Guideline Panels for Breast Cancer Screening and Diagnosis, and Breast Cancer Risk Reduction.

She is the recipient of many awards including the Julie and Ben Rogers Award for Excellence in Prevention in 2006, the Kathryn S. Stream Award for Excellence in Women's Health in 2011, and the Faculty Achievement Award in Prevention in 2011.

\section{Breast Cancer Risk Reduction Therapy: The Low-Hanging Fruit}

\author{
Therese B. Bevers, MD
}

More than 15 years ago, tamoxifen was FDA-approved for breast cancer risk reduction in women at an increased risk of developing breast cancer. Since that time, raloxifene has also received FDA approval for this purpose. Both of these drugs halve the risk of breast cancer while also reducing the risk of osteoporotic fractures, ${ }^{1,2}$ an indication for which raloxifene also has a FDA indication. The benefits of tamoxifen have been shown to have long-term durability, even after therapy is discontinued. ${ }^{3}$ In follow-up analyses, breast cancer risk reduction is greater with tamoxifen than with raloxifene, with raloxifene maintaining $75 \%$ of the benefits of tamoxifen after discontinuation of therapy. ${ }^{4}$ Clinical trials assessing the use of the aromatase inhibitors (AIs) exemestane and anastrozole have shown even greater breast cancer risk reduction for women at increased risk of developing the disease. ${ }^{5,6}$ However, the trials were small and these agents are not currently FDA-approved for this indication. Thus, their use for breast cancer risk reduction is currently considered "off-label" in the United States.

Unfortunately, risk reduction therapy has been vastly underused. Less than $0.25 \%$ of eligible women use these medications. ${ }^{7}$ In a 2003 analysis, Freedman et al ${ }^{8}$ noted that approximately 2.5 million American women aged 35 to 70 years could benefit from tamoxifen. Because risks would outweigh benefits for most women, their estimate represents only $25 \%$ of women in this age range who would be considered eligible for risk reduction therapy. Nonetheless, this indicates that a huge opportunity exists for these drugs to have a significant impact on the incidence of breast cancer.

In a recent analysis of prescribing patterns of primary care physicians (PCPs), only $13 \%$ of internists, family physicians, and gynecologists recommended or prescribed risk reduction therapy to their patients. ${ }^{9}$ From the perspective of most PCPs, the risks associated with the use of these agents were a major limiting factor. Both tamoxifen and raloxifene are associated with an increased risk of venous thromboembolic events (VTE), with raloxifene having a slightly lower risk than tamoxifen. ${ }^{2}$ In addition, tamoxifen is associated with an increased risk of endometrial cancer and cataracts; risks that are not increased with raloxifene. ${ }^{1,2}$ No serious risks have been identified in the prevention trials involving AIs.

It is critical that we help clinicians, especially those in the primary care setting, easily identify women for whom risk reduction therapy should be seriously considered. So who are these women?

Women for whom the benefits of risk reduction therapy almost always outweigh the harms are those with atypical hyperplasia $(\mathrm{AH})$, both ductal and lobular types, and the lobular carcinoma in situ (LCIS). Women with AH and LCIS have a significantly increased risk of developing breast cancer. Historical estimates of breast cancer risk range from a 4- to 5-fold increased risk in women with $\mathrm{AH}^{10}$ and an 8- to 10-fold increased risk in those with LCIS. ${ }^{11}$ Risk can be calculated using the Gail model (includes AH only, with limited family history) ${ }^{12}$ and the Tyrer-Cuzick model (includes both AH and LCIS, along with extensive family history). ${ }^{13}$ However, in an analysis by Mayo Clinic investigators, the Gail model was found to underestimate breast cancer risk for women with $\mathrm{AH},{ }^{14}$ whereas the Tyrer-Cuzick model overestimated the risk for this group of women. ${ }^{15}$ More recently, Hartmann et $\mathrm{al}^{16}$ suggested that women with $\mathrm{AH}$ have an absolute risk of $1 \%$ per year of developing breast cancer. Coopey et a $\mathrm{l}^{17}$ noted that the 10 -year risk is $17.3 \%$ for women with atypical ductal hyperplasia,
The ideas and viewpoints expressed in this editorial are those of the author and do not necessarily represent any policy, position, or program of NCCN. 
20.7\% for those with atypical lobular hyperplasia, and $23.7 \%$ for those with LCIS, even greater than the $1 \%$ risk per year identified by the Mayo cohort. The takehome message is that women with AH or LCIS are at a significantly increased risk of developing breast cancer!

The Breast Cancer Prevention Trial (P-1) showed that women with AH and LCIS obtain greater breast cancer risk reduction with tamoxifen than other women at high risk. The P-1 trial showed a significant $86 \%$ risk reduction for women with $\mathrm{AH}$ and a statistically nonsignificant $56 \%$ breast cancer risk reduction for women with LCIS at the initial analysis. ${ }^{1}$ Follow-up analyses continue to show a significant risk reduction of $75 \%$ for women with AH. Women with LCIS had a statistically nonsignificant $46 \%$ breast cancer risk reduction. ${ }^{18}$ Nonetheless, as a result of the extremely high risk of developing breast cancer that is associated with LCIS, the risk reduction for women with this disorder can be significant. The Study of Tamoxifen and Raloxifene (STAR) trial $^{2}$ showed no significant difference in the therapeutic effect of raloxifene compared with tamoxifen in women with AH and LCIS while on therapy.

The data on exemestane and anastrozole in women with AH or LCIS are limited. In this subgroup, anastrozole reduced breast cancer risk by $69 \%$ (hazard ratio [HR], $0.31 ; 95 \% \mathrm{CI}, 0.12-0.84),{ }^{6}$ whereas exemestane produced a nonsignificant reduction in the risk of breast cancer by $64 \%(\mathrm{HR}, 0.36$; $95 \% \mathrm{CI}, 0.11-1.12) .{ }^{5}$ Importantly, these analyses were in a very small number of women, which limits the ability to assess the effectiveness of these therapies in women with LCIS or AH. Although both AIs are options for breast cancer risk reduction, currently they may not be the best choice for women with proliferative breast lesions, given the significantly greater risk reduction that has been demonstrated for women with selective estrogen receptor modulators (SERMs). Further analyses of these agents in women with AH and LCIS is needed to better guide clinical decision-making.

Women with AH or LCIS are optimal candidates for preventive therapy. The benefits of these agents are considerable, because this population has an extremely high breast cancer risk and can obtain substantial breast cancer risk reduction from therapy. Unfortunately, in an analysis by Coopey et $a l,{ }^{17}$ fewer than one-fifth of women with these lesions were documented to have received risk reduction therapy. Although women on SERMs may experience serious adverse events, the harms are small compared with the significant benefits obtained in this population. In the absence of known absolute contraindications (eg, prior VTE in women considering tamoxifen or raloxifene), women with AH or LCIS should be started on risk reduction therapy.

The NCCN Breast Cancer Risk Reduction Panel added the following footnote in the 2015 guideline update: "Women with AH have an 86\% reduction in risk with therapy. Risk reduction therapy should be strongly recommended." ${ }^{19}$

The potential of bothersome side effects such as hot flashes should not prevent the start of therapy. Most women tolerate therapy with minimal if any symptoms. Those experiencing significant side effects should be appropriately managed. If symptoms continue to adversely impact quality of life, reconsidering whether therapy is appropriate for an individual woman is certainly appropriate. However, the potential for adverse events should not preclude prescribing risk reduction therapy in this very high-risk population. In other words, let's not anticipate side effects. Let's see if the patient actually develops side effects that limit the use of risk reduction therapy!

There is an imperative need to guide PCPs to understand that there are women at a significantly increased risk of breast cancer for whom benefits far outweigh harms and who should be on risk reduction therapy. I challenge everyone reading this: Are you prescribing risk reduction therapy for your patients with AH or LCIS? If not, why not? More importantly, we need to get PCPs to understand that prescribing risk 
Bevers

reduction therapy in women with AH or LCIS should be as automatic as prescribing antihypertensive medication for individuals presenting with extremely high blood pressure. They shouldn't leave the clinic without a prescription!

\section{References}

1. Fisher B, Costantino JP, Wickerham DL, et al. Tamoxifen for prevention of breast cancer: report of the National Surgical Adjuvant Breast and Bowel Project P-1 study. J Natl Cancer Inst 1998;90:1371-1388.

2. Vogel VG, Costantino JP, Wickerham DL, et al. Effects of tamoxifen vs raloxifene on the risk of developing invasive breast cancer and other disease outcomes: the NSABP Study of Tamoxifen and Raloxifene (STAR) P-2 trial. JAMA 2006;295:2727-2741.

3. Cuzick J, Sestak I, Cawthorn S, et al. Tamoxifen for prevention of breast cancer: extended long-term follow-up of the IBIS-I breast cancer prevention trial. Lancet Oncol 2015;16:67-75.

4. Vogel VG: Costantino JP,Wickerham DL, et al. Update of the NSABP Study of Tamoxifen and Raloxifene (STAR) P-2 trial: preventing breast cancer. Cancer Prev Res 2010;3:696-706.

5. Goss PE, Ingle JN, Alés-Martínez JE, et al. Exemestane for breast-cancer prevention in postmenopausal women. N Engl J Med 2011;364: 2381-2391.

6. Cuzick J, Sestak I, Forbes JF, et al. Anastrozole for prevention of breast cancer in high-risk postmenopausal women (IBIS-II): an international, double-blind, randomized placebo-controlled trial. Lancet 2014;383:1041-1048.

7. Waters EA, McNeel TS, Stevens WM, Freedman AN. Use of tamoxifen and raloxifene for breast cancer chemoprevention in 2010. Breast Cancer Res Treat 2012;134:875-880.

8. Freedman AN, Graubard BI, Rao SR, et al. Estimates of the number of U.S. women who could benefit from tamoxifen for breast cancer chemoprevention. J Natl Cancer Inst 2003;95:526-532.

9. Corbelli J, Borrero S, Bonnema R et al. Use of the Gail model and breast cancer preventive therapy among three primary care specialities. J Womens Health 2014;23:746-752.

10. Dupont WD, Page DL. Risk factors for breast cancer in women with proliferative breast disease. N Engl J Med 1985;312:146-151.

11. Page DL, Kidd TE Jr, Dupont WD, et al. Lobular neoplasia of the breast: higher risk for subsequent invasive cancer predicted by more extensive disease. Hum Pathol 1991;22:1232-1239.

12. Gail MH, Brinton LA, Byar DP, et al. Projecting individualized probabilities of developing breast cancer in white females who are being examined annually. J Natl Cancer Inst 1989;81:1879-1886.

13. Tyrer J, Duffy SW, Cuzick J. A breast cancer prediction model incorporating familial and personal risk factors. Stat Med 2004;23:1111-1130.

14. Pankratz VS, Hartmann LC, Degnim AC, et al. Assessment of the accuracy of the Gail model in women with atypical hyperplasia. J Clin Oncol 2008;26:5374-5379.

15. Boughey JC, Hartmann LC, Anderson SS, et al. Evaluation of the Tyrer-Cuzick (International Breast Cancer Intervention Study) model for breast cancer risk prediction in women with atypical hyperplasia. J Clin Oncol 2010;28:3591-3596.

16. Hartmann LC, Radisky DC, Frost MH, et al. Understanding the premalignant potential of atypical hyperplasia through its natural history: a longitudinal cohort study. Cancer Prev Res (Phila) 2014;7:211-217.

17. Coopey SB, Mazzola E, Buckley JM, et al. The role of chemoprevention in modifying the risk of breast cancer in women with atypical breast lesions. Breast Cancer Res Treat 2012;136:627-633.

18. Fisher B, Costantino JP, Wickerham DL, et al. Tamoxifen for the prevention of breast cancer: current status of the National Surgical Adjuvant Breast and Bowel Project P-1 study. J Natl Cancer Inst 2005;97:1652-1662.

19. Bevers TB, Ward JH, Arun BK, et al. NCCN Clinical Practice Guidelines in Oncology for Breast Cancer Risk Reduction, Version 1.2015; in press. 